\title{
Stress analysis of double-walled pipes undergone mechanical drawing process
}

\author{
RuoGu Zhang ${ }^{1}$ (1) $\cdot$ Yu Wang ${ }^{1} \cdot$ Yinbo Zhu ${ }^{1} \cdot$ JiDong Jin $^{1} \cdot$ HengAn Wu ${ }^{1} \cdot$ Ping Gu $^{1} \cdot$ Yang Zhao $^{1,2}$
}

Received: 7 May 2021 / Accepted: 23 October 2021 / Published online: 1 January 2022

(c) The Author(s) 2021

\begin{abstract}
Double-walled metal pipes are important components for heat exchange and liquid transportation. They can be manufactured by mechanical drawing of two concentric pipes. In this work, a mechanical model is developed to analyze the stress state evolution during the manufacturing process, and the criterion for forming the double-walled pipe was given under the ideal elastoplastic and thick-walled assumptions. The model also encompasses the results deduced under the thin-walled assumption. Numerical simulations confirmed that the accuracy of the analytical model was within 5\%. The application on actual steel materials with various parameters varied, including the wall thickness and initial gap, was analyzed. This work can provide theoretical support to industrial manufacturing procedures and help to reduce costs by eliminating required test procedures.
\end{abstract}

Keywords Double-walled pipe $\cdot$ Residual stress $\cdot$ Elastoplasticity $\cdot$ Numerical simulation

\section{Introduction}

Double-walled pipes are widely used in various applications, such as applications in the chemical industry and nuclear power plants, because of their ability to cope with extreme temperature differences or chemical environments inside and outside the pipes and effectively prevent crack propagation through the pipes [1]. Double-walled pipes consist of two layers made of the same or different kinds of materials with or without an interlayer [2-5]. Directly connected doublewalled pipes can provide a sufficient heat transfer efficiency

Ping Gu

guping@ustc.edu.cn

$\triangle$ Yang Zhao

yangzhao1@ustc.edu.cn

RuoGu Zhang

251213177@qq.com

1 Department of Modern Mechanics, CAS Key Laboratory of Mechanical Behavior and Design of Materials, University of Science and Technology of China, CAS Center for Excellence in Complex System Mechanics, Chinese Academy of Science, Hefei 230027, China

2 Macro \& Nano Thermal Science Laboratory, Department of Precision Machinery \& Precision Instrumentation, University of Science and Technology of China, 230027 Hefei, China and prevent liquid leakage caused by rupture. Thus, they are very useful in the heat exchange equipment of nuclear reactors.

The double-walled pipe can be manufactured by various methods, such as welding, explosion-based methods, or mechanical methods to press two layers together. Most previous research focused on the processing parameters for the manufacturing procedures [1, 6-10, 16]. Chen [7] summarized some existing processing techniques. Kamal and Dixit [16] selected a thermal autofrettaging method and combined it with a shrink-fitting method. Delong [8] made composite double-walled pipes by using hot extrusion first and then cold drawing. Bogatov [9] fabricated double-walled pipes under an internal pressure that could be used in the petroleum industry. Taheri-Behrooz and Pourahmadi [10] studied the effects of high temperatures on the mechanical performances of double-walled pipes.

Theoretical analyses of the stress and strain states of double-walled pipes during the manufacture procedures have been performed. Kim [11] proposed a method to measure residual stresses in double-walled pipes using neutron diffraction technology and verified the results with calculations. Wang and $\mathrm{Li}$ [12] discussed the stress and strain state of a pipe made using a hydraulic expansion method and provided suitable processing parameters. Lv 
[13] ignored the thickness of the inner pipe. Jia [14] considered both layers to be thin walls and presented criteria for the mechanical manufacturing of double-walled pipes. Although the thickness of the wall is generally small relative to the pipe diameter, the thick-walled assumption is worth considering because the thin-walled assumption cannot adequately represent the pipes used in real situations, and information related to the wall thickness allows us to study complex deformation and failure.

In this work, a detailed analysis on the stress state during mechanical manufacturing was performed based on the thick-walled and ideal elastoplastic assumptions. The results cover both thin- and thick-walled cases. The analytical model was validated by finite element numerical simulations. The criterion to successfully form double-walled pipes and the inner pressure exerted on the inner wall to obtain the maximum residual stress are presented. To meet the needs of actual manufacturing processes, adjustments to the theory were also presented based on the properties of some common industrial materials.

\section{Analytical model of forming double-walled pipes by mechanical methods}

The schematic diagram of the mechanical drawing process is shown in Fig. 1. The mechanical drawing method can be grouped into internal extrusion and external pressing methods. The analysis procedures of these two methods are similar. In our work, the internal extrusion method is selected as an example, and the internal load in the mechanical drawing procedure is simplified as a uniform internal pressure.
The analysis is divided into the following steps: (1) identification of the stress and strain in a single-walled pipe under an internal pressure, (2) identification of the stress and strain in the inner shell of the two concentric pipes before they make contact, (3) the change of the stress in the doublewalled pipe after the two shells make contact (unloading will start at the end of this period), (4) evolution of the stress and strain of the pipe as it undergoes the unloading procedure, and (5) evaluation of the residual pressure between the shells after unloading. The existence of residual stress is regarded as the criterion for successful manufacturing of double-walled pipes.

\subsection{Stress and strain analysis of single-walled pipe}

When a single-walled pipe is subjected to an increasing internal or external radial pressure, it will gradually enter the plastic state from the innermost surface to the outmost surface. A plastic interface will emerge in the pipe wall, inside which there is a plastic zone, and outside there is an elastic zone. It will expand outwards. When it reaches the outermost surface, the entire pipe enters the plastic regime [3]. The evolution of the stress and strain of a double-walled pipe will be similar based on the expansion of the plastic interface.

Based on plane strain model, the stress and strain analysis of the mechanical internal extrusion method is carried out using the Tresca stress condition and the ideal elastic-plastic hypothesis. For a pipe with inner and outer radii $a$ and $b$, internal pressure $q_{1}$, and external pressure $q_{2}=0$, there is a plastic interface at radius $r_{p}$, and the radial stress at this interface is $q_{p}$, as shown in Fig. 2a. The interior zone of the interface is plastic, while the exterior zone is elastic. The radial and tangential stress distribution can be expressed as
Fig.1 a Manufacturing process of mechanical drawing method, equivalent to the internal pressure method with determined displacement [17]. b Schematic diagram of internal extrusion method (the spacing between the two pipes will disappear after processing)
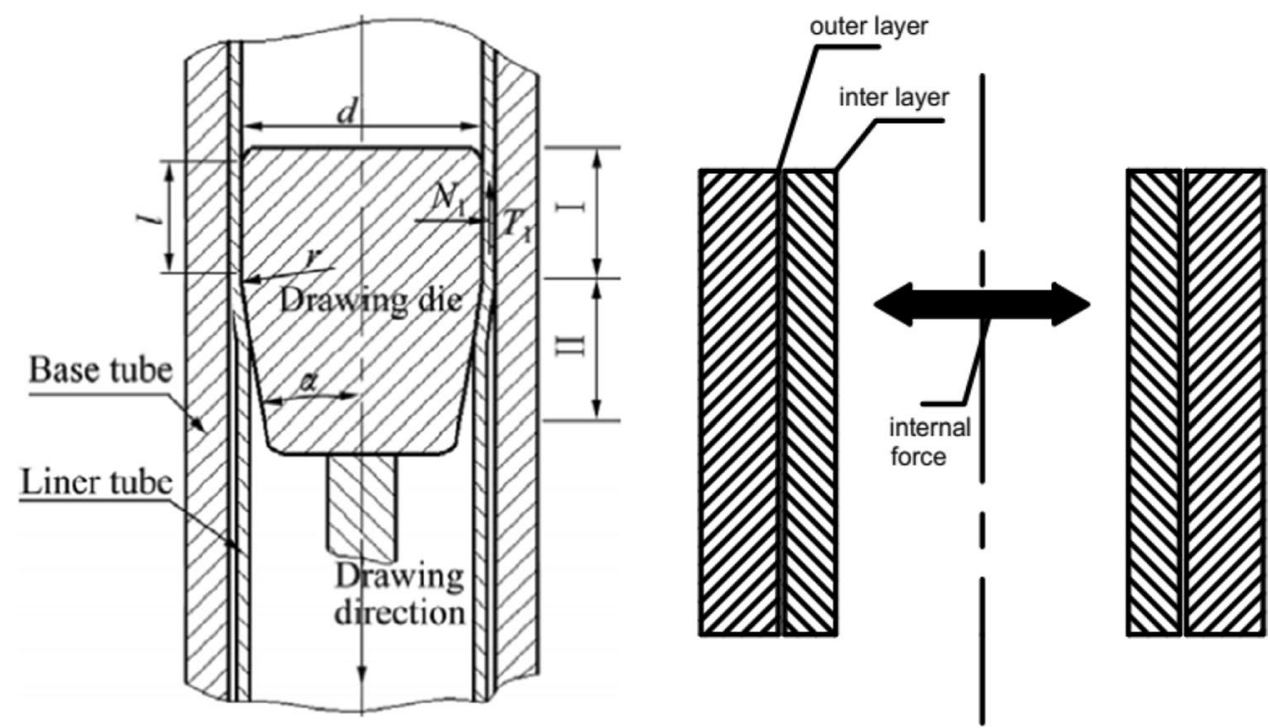

(b) 
Fig. 2 a Schematic diagram of a single-walled pipe. b Schematic diagram of a double-walled pipe

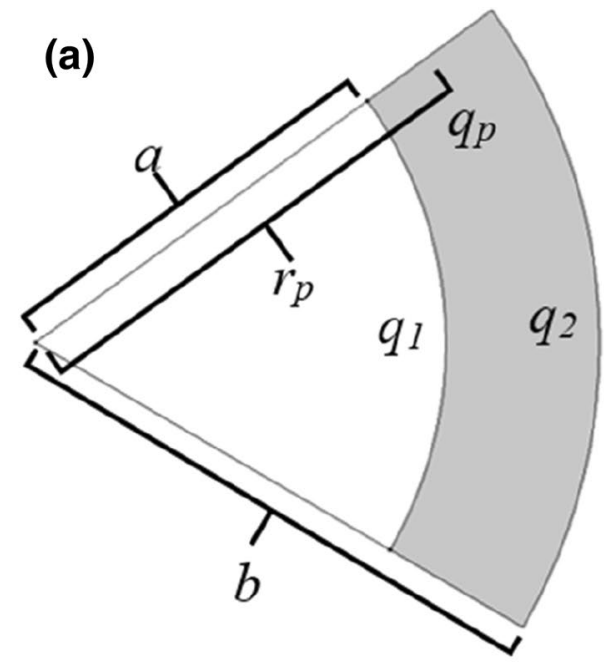

$\sigma_{r}=\left\{\begin{array}{cc}\sigma_{s} \ln \frac{r}{a}-q_{1} & a \leq r \leq r_{p} \\ \left(1-\frac{b^{2}}{r^{2}}\right) \frac{r_{p}^{2} q_{p}}{b^{2}-r_{p}^{2}} & r_{p} \leq r \leq b\end{array}\right.$,

$\sigma_{\theta}=\left\{\begin{array}{cc}\sigma_{s}\left(\ln \frac{r}{a}+1\right)-q_{1} & a \leq r \leq r_{p} \\ \left(1+\frac{b^{2}}{r^{2}}\right) \frac{r_{p}^{2} q_{p}}{b^{2}-r_{p}^{2}} & r_{p} \leq r \leq b\end{array}\right.$,

where $\sigma_{s}$ is the yield stress of the pipe.

The distribution of the displacement along the radius can be expressed as follows:

$u=\left\{\begin{array}{ll}\frac{1}{2} \frac{(1+v) \sigma_{s} r_{p}^{2}}{E b^{2} r}\left[(1-2 v) r_{p}^{2}+b^{2}\right] & a \leq r \leq r_{p} \\ \frac{1}{2} \frac{(1+v) \sigma_{s} r_{p}^{2}}{E b^{2} r}\left[(1-2 v) r^{2}+b^{2}\right] & r_{p} \leq r \leq b\end{array}\right.$.

If the whole pipe is still in the elastic region, then

$u=\frac{(1+v) q_{1} a^{2}}{E\left(b^{2}-a^{2}\right)}\left[(1-2 v) r+\frac{b^{2}}{r}\right]$.

Similarly, the stress distribution under an external pressure of $q_{2}$ (while $q_{1}=0$ ) can be obtained:

$\sigma_{r}=\left\{\begin{array}{cc}\frac{r_{p}^{2} b^{2}\left(q_{2}-q_{p}\right)}{b^{2}-r_{p}^{2}} \frac{1}{r^{2}}+\frac{r_{p}^{2} p_{p}-b^{2} q_{2}}{b^{2}-r_{p}^{2}} & a \leq r \leq r_{p}, \\ \sigma_{s} \ln \frac{r}{a} & r_{p} \leq r \leq b\end{array}\right.$
$\sigma_{\theta}=\left\{\begin{array}{cc}-\frac{r_{p}^{2} b^{2}\left(q_{2}-p_{p}\right)}{b^{2}-r_{p}^{2}} \frac{1}{r^{2}}+\frac{r_{p}^{2} p_{p}-b^{2} q_{2}}{b^{2}-r_{p}^{2}} & a \leq r \leq r_{p} \\ \sigma_{s}\left(\ln \frac{r}{a}+1\right) & r_{p} \leq r \leq b\end{array}\right.$.
It is worth noting that, as can be seen from here, even if the pipe is under an external pressure, it will also enter the plastic state from the inner surface.

The displacement along the radius is

$u=\left\{\begin{array}{cc}-\frac{1}{2} \frac{(1+v) \sigma_{s} r_{p}^{2}}{E a^{2} r}\left[(1-2 v) r^{2}+a^{2}\right] & a \leq r \leq r_{p} \\ -\frac{1}{2} \frac{(1+v) \sigma_{s} r_{p}^{2}}{E a^{2} r}\left[(1-2 v) r_{p}^{2}+a^{2}\right] & r_{p} \leq r \leq b\end{array}\right.$.

\subsection{Stress and strain analysis of double-walled pipe}

\subsubsection{Contact of two layers of a double-walled pipe}

The inner radius of the inner pipe is $a$, the outer radius of the outer pipe is $c$, and the initial gap between the inner and outer pipe is $d_{0}$, as shown in Fig. 2b. Young's modulus, Poisson's ratio, and ultimate stress of the material are denoted as $\mathrm{E}, \nu$, and $\sigma$, respectively, and the inner and outer pipes are denoted by the subscripts 1 and 2, respectively. Since the spacing between the pipes is much smaller than the radius of the pipes, the outer radius of the inner pipe and the inner radius of the outer pipe both can be regarded as $b$.

According to the study of a single pipe (Eq. 7), the stress and strain states of the inner pipe when the pipes make contact will be determined by the initial gap between two layers. The different states are the elastic-deformed state, completely plastic-deformed state, or a state in between.

1. When the pipes make contact, $r_{p}=a$, and $\left.u\right|_{r=b}=u_{e}$. The second formula of Eq. (7) can be written as

$u_{e}=\frac{1}{2} \frac{\left(1+v_{1}\right) \sigma_{s 1} a^{2}}{E_{1} b^{3}}\left[\left(1-2 v_{1}\right) b^{2}+b^{2}\right]=\frac{\left(1-v_{1}^{2}\right) \sigma_{s 1} a^{2}}{b E_{1}}$. 
If $d_{0} \leq u_{e}$, the inner pipe is still in an elastic state when two layers make contact.

2. When the pipes make contact, $r_{p}=b$, and $\left.u\right|_{r=b}=u_{l}$. The first formula of Eq. (8) can be written as

$u_{l}=\frac{1}{2} \frac{\left(1+v_{1}\right) \sigma_{s 1} b^{2}}{E_{1} b^{3}}\left[\left(1-2 v_{1}\right) b^{2}+b^{2}\right]=\frac{\left(1-v_{1}^{2}\right) \sigma_{s 1} b}{E_{1}}$.

If $d_{0} \geq u_{e}$, the inner pipe is completely in the plastic regime when two pipes make contact.

3. When $d_{0}$ is between $u_{e}$ and $u_{l}$, there will be a plastic interface in the inner pipe as the pipes contact. The radius of the plastic interface is $r_{p 1}$.

\subsubsection{After two layers contact}

The internal pressure of the surface where the inner and outer pipes contact is denoted as $q_{r}$. If the inner pipe is still in the elastic stage when two pipes make contact (Eq. 9), the conditions for the inner surface of the pipes to enter the plastic regime can be written as

$q_{1}-q_{r}=\frac{b^{2}-a^{2}}{2 b^{2}} \sigma_{s 1}($ inner pipe),

$q_{r}=\frac{c^{2}-b^{2}}{2 c^{2}} \sigma_{s 2}($ outer pipe $)$

If Eq. (10) is satisfied before Eq. (11), the inner pipe will enter the plastic regime earlier than the outer pipe. On the contrary, the outer pipe will enter the plastic regime earlier.

The condition for when the outer pipe enters the full plastic regime is as follows:

$q_{r}=\sigma_{s 2} \ln \frac{c}{b}$,

and condition for when the inner pipe enters the full plastic regime is as follows:

$q_{1}-q_{1}^{\prime}=\sigma_{s 1} \ln \frac{b}{a}$,

where $q_{1}^{\prime}$ indicates the increment of $q_{1}$ that occurs as the pressure $q_{r}$ is applied on the interfaces between inner and outer pipes. $q_{1}$ cannot increase after the inner pipe completely enters the plastic stage. However, when the outer pipe obstructs the deformation process of the inner pipe, $q_{1}^{\prime}$ and $q_{r}$ will appear on the inner and outer surfaces of the inner pipe. $q_{r}$ undergoes reverse elastic loading on the inner pipe, so $q_{1}^{\prime}$ offsets $q_{r}$ if the effects of the wall thickness are ignored.

In the actual production process, the gap between the inner and outer pipes is sufficiently large that the inner pipe is already in the full plastic regime when contact is made. This will be the default assumption in later sections.

\subsubsection{Unloading}

After contact, the unloading from any of the stress states mentioned in Sect. 1.2.2 can be calculated by the following method. Some materials have a lag in deformation, and the load must be held for a period of time to ensure that sufficient deformation occurs. Considering that some materials have a lag in deformation because of viscoelasticity, it is necessary to maintain the load for a period to ensure sufficient deformation [15]. This is not considered in this article.

The unloading process is an elastic process. For unloading stresses of $\Delta q_{1}$ at $r=a$, and $\Delta q_{r}$ at $r=b$,

$\Delta \sigma_{r}=\left\{\begin{array}{cc}\frac{a^{2} b^{2}\left(\Delta q_{r}-\Delta q_{1}\right)}{\left(b^{2}-a^{2}\right) r^{2}}+\frac{a^{2} \Delta q_{1}-b^{2} \Delta q_{r}}{b^{2}-a^{2}} & a \leq r \leq b \\ -\frac{b^{2} \Delta q_{r}}{c^{2}-b^{2}}\left(\frac{c^{2}}{r^{2}}-1\right) & b \leq r \leq c\end{array}\right.$,

$\Delta \sigma_{\theta}=\left\{\begin{array}{cl}-\frac{a^{2} b^{2}\left(\Delta q_{r}-\Delta q_{1}\right)}{\left(b^{2}-a^{2}\right) r^{2}}+\frac{a^{2} \Delta q_{1}-b^{2} \Delta q_{r}}{b^{2}-a^{2}} & a \leq r \leq b \\ \frac{b^{2} \Delta q_{r}}{c^{2}-b^{2}}\left(\frac{c^{2}}{r^{2}}+1\right) & b \leq r \leq c\end{array}\right.$.

The radial strain condition is

$\left.\Delta \varepsilon_{r 1}\right|_{r=b}=\left.\Delta \varepsilon_{r 2}\right|_{r=b}$.

Thus,

$\frac{\nu_{1}\left(2 a^{2} \Delta q_{1}-\left(a^{2}+b^{2}\right) \Delta q_{r}\right)}{\left(b^{2}-a^{2}\right) E_{1}}+\frac{\Delta q_{r}}{E_{1}}=\frac{\nu_{2}\left(c^{2}+b^{2}\right)}{c^{2}-b^{2}} \frac{\Delta q_{r}}{E_{2}}+\frac{\Delta q_{r}}{E_{2}}$.

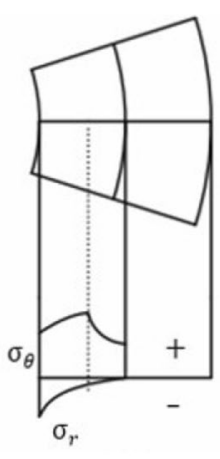

(a)

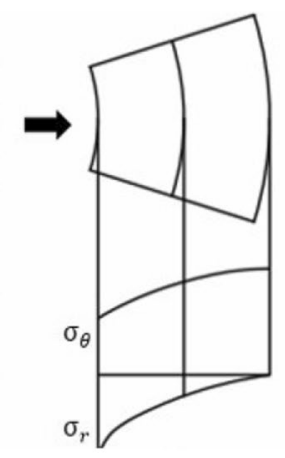

(b)

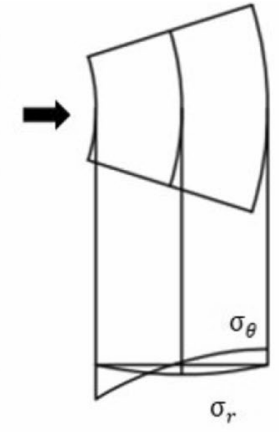

(c)
Fig. 3 Schematic diagram of the internal pressure loading process: a the inner and outer pipes are not in contact during loading (the dotted line is the elastoplastic boundary), $\mathbf{b}$ after loading and both the inner and outer pipes are completely plastic, and $\mathbf{c}$ after unloading, residual compressive stress exists between the two pipes 
When $\Delta q_{1}=q_{1}$, the load is completely removed. If $\Delta q_{r} \leq q_{r}$, the pipes can be pressed together; otherwise, they will be disconnected. The residual compressive stress between the two pipes is $q_{r e}=q_{r}-\Delta q_{r}$. When $\Delta q_{r}=q_{r}$, the inner and outer pipes just fit together, but there is no pressure on the contact surface.

\subsection{Criterion for successful manufacturing of double-walled pipe}

The inner pipe is already full in the plastic regime when contact is made: $q_{1}=\sigma_{s 1} \ln \frac{b}{a}+q_{r}$

When $\Delta q_{1}=q_{1}$, we obtain

$\Delta q_{1}=q_{r}\left[\frac{1}{E_{2}}\left(v_{2} \frac{c^{2}+b^{2}}{c^{2}-b^{2}}+1\right)+\frac{1}{E_{1}}\left(v_{1} \frac{a^{2}+b^{2}}{b^{2}-a^{2}}-1\right)\right] \frac{b^{2}-a^{2}}{2 a^{2} v_{1}} E_{1}$.

We let

$$
B \equiv\left[\frac{1}{E_{2}}\left(v_{2} \frac{c^{2}+b^{2}}{c^{2}-b^{2}}+1\right)+\frac{1}{E_{1}}\left(v_{1} \frac{a^{2}+b^{2}}{b^{2}-a^{2}}-1\right)\right] \frac{b^{2}-a^{2}}{2 a^{2} v_{1}} E_{1},
$$

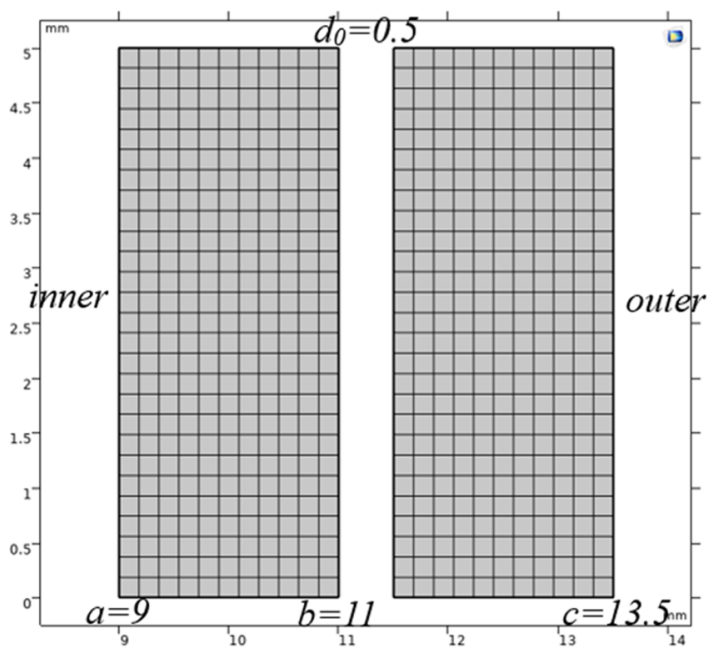

(a)

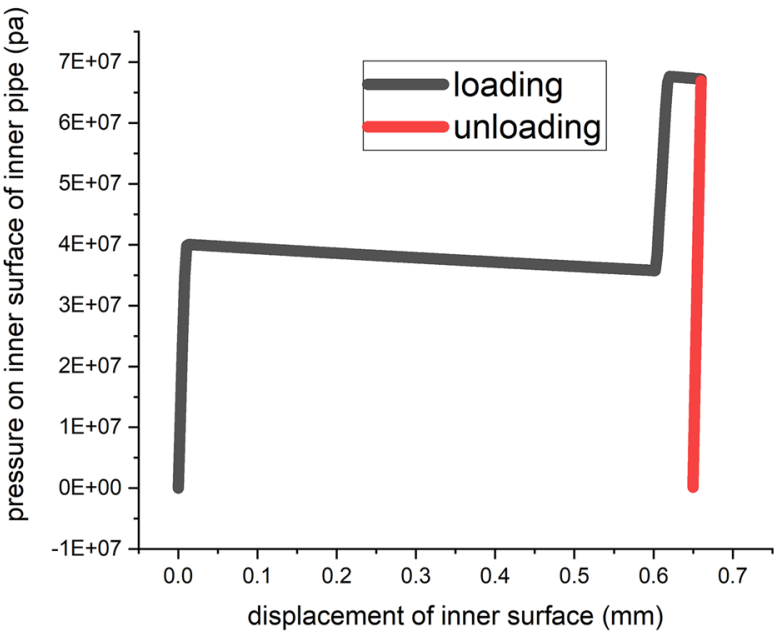

(b)

Fig. 4 a Model used for numerical simulation. b Numerical simulation results of the relationship between the displacement of the inner surface and the pressure on the innermost surface of the inner pipe.

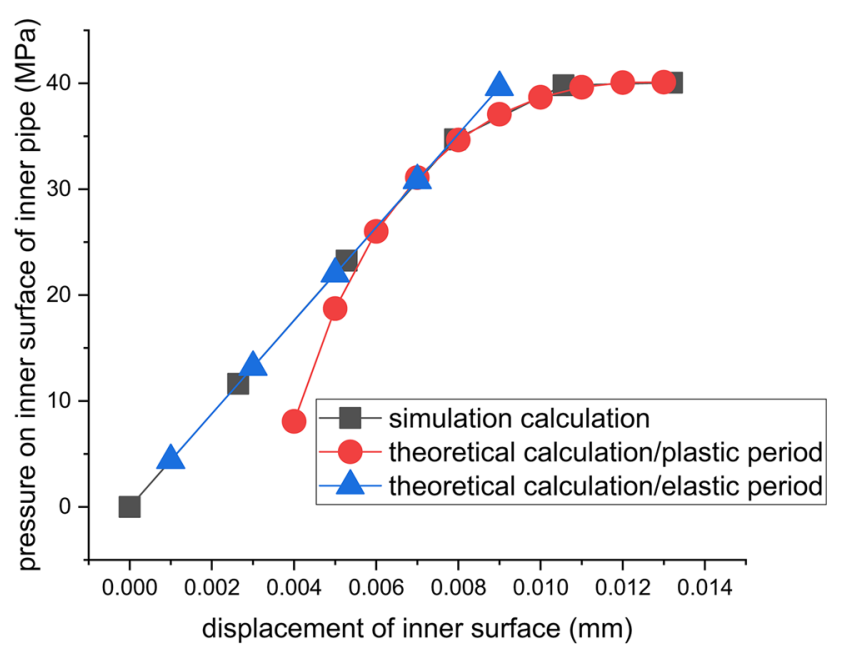

(c)

The region in the circle corresponds to Fig. 4c. c Agreement between the theory and the simulation results 
and thus,

$(B-1)\left(q_{1}-\sigma_{s 1} \ln \frac{b}{a}\right)>\sigma_{s 1} \ln \frac{b}{a}$.

If Eq. (25) is not satisfied, the compacted double-walled pipe cannot be obtained. In this case, the following can be used as a criterion:

$(B-1) \frac{\sigma_{s 2} \ln \frac{c}{b}}{\sigma_{s 1} \ln \frac{b}{a}}>1$.

The residual stress is

$q_{r e}=q_{r}-\frac{q_{1}}{B}$.

When $q_{1}=q_{1 \max }=\sigma_{s 1} \ln \frac{b}{a}+\sigma_{s 2} \ln \frac{c}{b}$ (both pipes enter fully plastic regimes before unloading), $q_{r e}$ will reach a maximum:

$q_{\text {re-max }}=\sigma_{s 2} \ln \frac{c}{b}-\frac{1}{B}\left(\sigma_{s 1} \ln \frac{b}{a}+\sigma_{s 2} \ln \frac{c}{b}\right)$.

If the pipes are formed by an external pressure, the criterion can be obtained by a similar method (the outer pipe has entered the fully plastic regime before unloading):

$\frac{\sigma_{s 1} \ln \frac{b}{a}}{\sigma_{s 2} \ln \frac{c}{b}+\sigma_{s 1} \ln \frac{b}{a}} B_{2}>1$,

where

$B_{2} \equiv\left[\frac{1}{E_{2}}\left(v_{2} \frac{c^{2}+b^{2}}{c^{2}-b^{2}}-1\right)+\frac{1}{E_{1}}\left(v_{1} \frac{a^{2}+b^{2}}{b^{2}-a^{2}}+1\right)\right] \frac{c^{2}-b^{2}}{2 c^{2} v_{2}} E_{2}$.

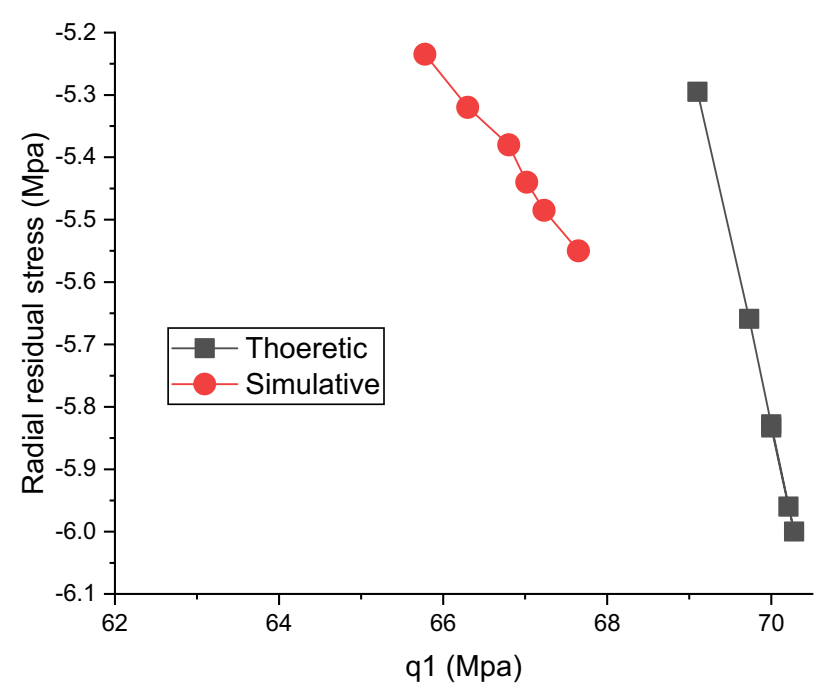

(a)
The residual stress is related to the thicknesses of the pipes. The final stress-strain distribution is shown in Fig. 3c. If there is a requirement on the minimum value of the residual stress in a real application, the initial thicknesses of the pipes can be specially selected using Eq. (28).

Under the thin-walled assumption, $a \approx b \approx c$ and $\ln \frac{b}{a} \approx \ln \frac{c}{b} \neq 0$. Therefore, $B \approx \frac{E_{1} v_{2}}{E_{2} v_{1}}+1$, and the criterion to press them together is $\frac{\sigma_{s 2} v_{2}}{E_{2}} \geq \frac{\sigma_{s 1} v_{1}}{E_{1}}$ (or $\varepsilon_{s 2} v_{2} \geq \varepsilon_{s 1} v_{1}$ ). Similarly, $B_{2} \approx \frac{E_{2} v_{1}}{E_{1} v_{2}}+1$ in the external pressure method, and the condition becomes $\frac{\sigma_{s 2} v_{2}}{E_{2}} \leq \frac{\sigma_{s 1} v_{1}}{E_{1}}$. When Poisson's ratios are considered to be the same for the inner and outer pipes, the results show agreement with previous work [6]. In this case, the inner and outer pipes made of the same material can only be pressed together with no pressure on the contact surface, and a sufficiently tight double-walled pipe cannot be successfully fabricated. The result deduced from the thick-walled assumption shows that residual stress can exist in this case, which is more realistic.

\subsection{Application in fabrication of stainless-steel double-walled pipe}

Data were chosen from the most popular commercial stainless-steel pipes, with $\sigma_{s}=200 \mathrm{MPa}, E=200 \mathrm{GPa}$, and $v=0.25$. The material is considered an ideal elastic-plastic model. The diameters of the outer and inner pipes were 27 and $22 \mathrm{~mm}$, while both wall thicknesses were $2 \mathrm{~mm}$. Referring to Fig. 2b, the parameters can be set as $a=9 \mathrm{~mm}$, $b=11 \mathrm{~mm}$, and $d_{0}=0.5 \mathrm{~mm}$ for the inner pipe before

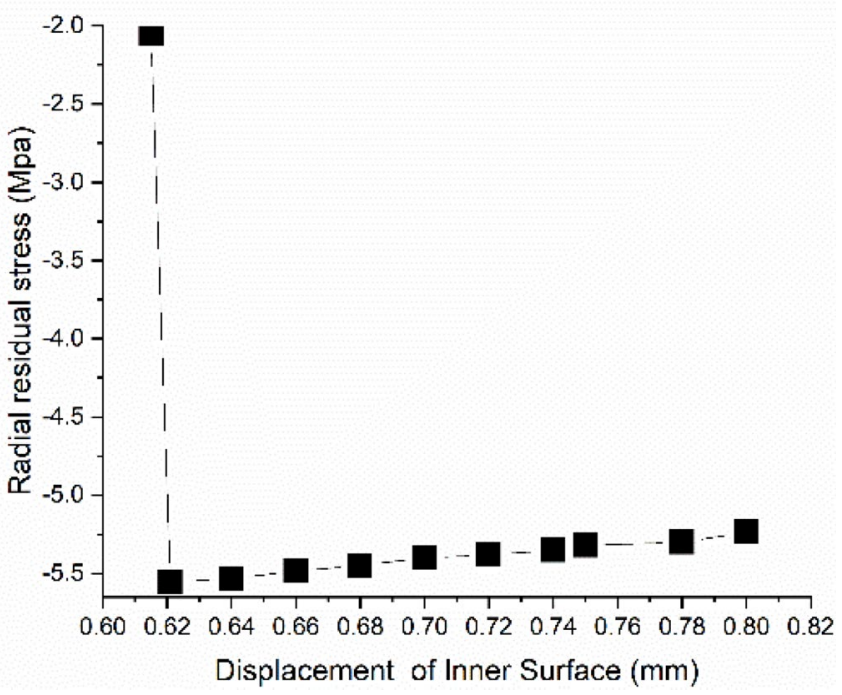

(b)

Fig.5 a Radial stress on the interface for different $q_{1}$. b Radial stress from displacement of the inner surface 
Table 1 Stresses of inner tube

\begin{tabular}{lll}
\hline & Inner wall of inner pipe & $\begin{array}{l}\text { Outer wall } \\
\text { of inner } \\
\text { pipe }\end{array}$ \\
\hline$\sigma_{r}^{\prime}$ & $-q_{1}^{\prime}$ & $-q_{r}$ \\
$\sigma_{\boldsymbol{\theta}}^{\prime}$ & $\frac{\left(a^{2}+b^{2}\right) q_{1}^{\prime}-2 b^{2} q_{r}}{b^{2}-a^{2}}$ & $\frac{2 a^{2} q_{1}^{\prime}-\left(a^{2}+b^{2}\right) q_{r}}{b^{2}-a^{2}}$ \\
\hline
\end{tabular}

the contact happens, and $a=9.5 \mathrm{~mm}, b=11.5 \mathrm{~mm}$, and $c=13.5 \mathrm{~mm}$ after contact.

If the inner pipe is still fully elastic when contact is made, the following must be satisfied:

$d_{0} \leq \frac{\left(1-v^{2}\right) \sigma_{s} a^{2}}{b E}=0.0069(\mathrm{~mm})$.

This requirement is almost impossible to achieve in practice. If the inner pipe is already fully plastic when in contact, it requires.

$d_{0} \geq \frac{\left(1-v^{2}\right) \sigma_{s} b}{E}=0.0103(\mathrm{~mm})$

which means it is reasonable that the inner pipe is already fully plastic when contact is made. Furthermore, if

$(B-1) \frac{\sigma_{s 2} \ln \frac{c}{b}}{\sigma_{s 1} \ln \frac{b}{a}} \approx 1.43>1$,

then the conditions for double-walled pipe fabrication are satisfied.

When $r_{p}=c, q_{r}=\sigma_{s 2} \ln \frac{c}{b}=32.07 \mathrm{MPa}$ and $q_{1}=\sigma_{s 1}$ $\ln \frac{b}{a}+\sigma_{s 2} \ln \frac{c}{b}=70.28 \mathrm{MPa}$, the maximum residual stress of $6.00 \mathrm{MPa}$ can be achieved.

\section{Model validation}

\subsection{Finite element analysis simulations}

We used the solid mechanics module in the COMSOL Multiphysics 5.2 software with a triangular mesh to simulate quasi-static conditions. The size and material parameters of the pipes are the same as those in Sect. 1.4.
The model used for the numerical simulation is shown in Fig. 4a, and the parameters are indicated in Fig. 4a. As in the theoretical part, the pipe is considered an ideal elastic-plastic material. The upper and lower boundaries of the pipe are set as symmetric boundary conditions, and external force comes from the inner side. Stress changes at the contact of the two pipes will be considered.

During loading, the inner wall of the inner pipe moved $0.66 \mathrm{~mm}$ outward, and when unloading, the inner wall of the inner pipe moved back $0.011 \mathrm{~mm}$. Figure $4 \mathrm{a}$ shows the pressure on inner surface and the displacement of the inner pipe. (1) to (2) is the external deformation stage of the internal pipe until it enters full plasticity. (2) to (3) is the stage after the inner pipe enters full plasticity and before the pipes make contact, showing that in the simulation, the inner pipe reached full plasticity before the two pipes made contact. During the period from (2) to (3), the pressure on the inner surface of the inner pipe did not increase but slightly decreased due to the increase in the radius (this decrease was ignored in the theoretical calculations). (3) to (4) is the stage after the pipes made contact and the outer pipe entered the fully plastic regime, corresponding to the stage in which $q_{1}$ generated the increment $q_{1}^{\prime}$ to offset the increase in $q_{r}$ in the theoretical calculations. The increase in the pressure in this stage in Fig. 4a was $q_{1}^{\prime}$. The outer pipe reached full plasticity at (4), and unloading began at (5. (6) represents the end of unloading. Both pipes in this simulation entered the fully plastic regime before unloading, and the outer pipe had just entered it. The obtained residual stress will be approximately the maximum value if the compression is successful. In Fig. 4b, the simulation results are compared with the theoretical results derived from Eqs. (6), (7), and (8). The inner pipe began to yield when the displacement of the inner surface was $0.7 \mathrm{~mm}$.

Figure 5a shows the theoretical and simulated radial stresses on the interface for different $q_{1}$. The results of the previous section are also included. The maximum value is at the right end of the curve. In theory, the maximum internal pressure is maintained and cannot be increased. On the $\mathrm{Y}$-axis, the results were similar, and on the $\mathrm{X}$-axis, there were slight differences. The reasons for these differences will be explained in the next section. Figure $5 \mathrm{~b}$ shows the radial stress on the interface obtained for different $u$ versus the displacement of the inner surface.
Table 2 Value of stress at critical position and time of inner tube

\begin{tabular}{|c|c|c|c|c|}
\hline \multirow[b]{2}{*}{ Time point } & \multicolumn{2}{|l|}{$\sigma_{\mathrm{r}} / \mathrm{MPa}$} & \multicolumn{2}{|l|}{$\sigma_{\theta} / \mathrm{MPa}$} \\
\hline & $\begin{array}{l}\text { Inner wall of } \\
\text { inner pipe }\end{array}$ & $\begin{array}{l}\text { Outer wall of } \\
\text { inner pipe }\end{array}$ & $\begin{array}{l}\text { Inner wall of } \\
\text { inner pipe }\end{array}$ & $\begin{array}{l}\text { Outer wall } \\
\text { of inner } \\
\text { pipe }\end{array}$ \\
\hline Outer pipe began to be pressed & -35.765 & 0.23091 & 164.24 & 200.23 \\
\hline Outer pipe entered full plasticity & -67.652 & -31.805 & 132.35 & 168.19 \\
\hline
\end{tabular}


Table 3 Value of stress at critical position of inner pipe

\begin{tabular}{lll}
\hline & $\begin{array}{l}\text { Inner wall of inner pipe } \\
/ 10^{6} \mathbf{P a}\end{array}$ & $\begin{array}{l}\text { Outer wall } \\
\text { of inner pipe } \\
/ 10^{6} \mathbf{P a}\end{array}$ \\
\hline$\sigma_{r}^{\prime} / \mathrm{Pa}$ & -31.887 & -31.574 \\
$\sigma_{\theta^{\prime}} / \mathrm{Pa}$ & -31.89 & -32.04 \\
\hline
\end{tabular}

\subsection{Verification of approximation in theoretical analysis}

In the theoretical calculation section, the increment $q_{1}^{\prime}$ of $q_{1}$ caused by $q_{r}$ can be taken as $q_{1}^{\prime}=q_{r}$. The rationality of this approximation is as follows. The elastic effect of $q_{r}$ and $q_{1}^{\prime}$ on the inner tube caused increments of the stresses in the inner pipe $\sigma_{\theta}^{\prime}$ and $\sigma_{r}^{\prime}$, respectively, and the distributions are

$\sigma_{r}^{\prime}=\frac{a^{2} b^{2}\left(q_{r}-q_{1}^{\prime}\right)}{b^{2}-a^{2}} \frac{1}{r^{2}}-\frac{b^{2} q_{r}-a^{2} q_{1}^{\prime}}{b^{2}-a^{2}}$,

$\sigma_{\theta}^{\prime}=\frac{a^{2} b^{2}\left(q_{1}^{\prime}-q_{r}\right)}{b^{2}-a^{2}} \frac{1}{r^{2}}-\frac{b^{2} q_{r}-a^{2} q_{1}^{\prime}}{b^{2}-a^{2}}$

The values at the inner and outer walls are shown in Table 1.

The variations of $\sigma_{\theta}$ and $\sigma_{r}$ of the inner and outer walls of the inner pipe during the simulation from contact to the outer pipe entering the fully plastic regime are shown in Table 2. The data in Table 3 are the simulation results corresponding to Table 1 .

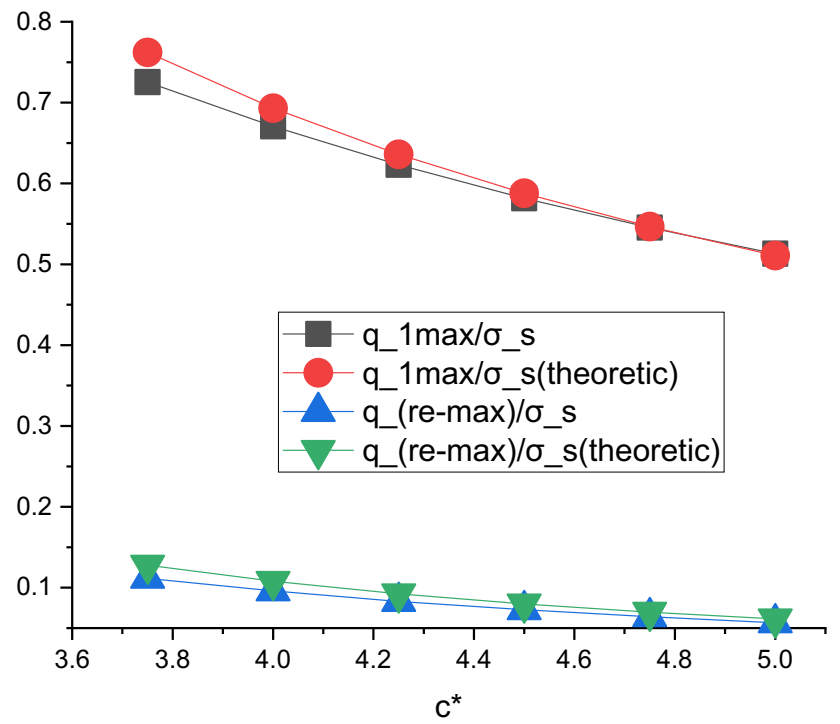

(a)
A $\mathrm{s}$ $\frac{\left(q_{1}^{\prime}-q_{r}\right)}{q_{1}^{\prime}}=\frac{\left(\sigma_{\text {rin }}^{\prime}-\sigma_{\text {rout }}^{\prime}\right)}{\sigma_{\text {rin }}^{\prime}}=\frac{-3.1887 \times 10^{7}-\left(-3.1574 \times 10^{7}\right)}{-3.1887 \times 10^{7}}=0.0098$, the difference between $q_{1}^{\prime}$ and $q_{r}$ is less than $1 \%$. If $q_{1}^{\prime}=q_{r}$ is obtained, the following can also be obtained:

$\sigma_{\theta \text { in }}^{\prime}=\sigma_{\theta o u t}^{\prime}=\frac{\left(a^{2}+b^{2}\right) q_{1}^{\prime}-2 b^{2} q_{r}}{b^{2}-a^{2}}=\frac{2 a^{2} q_{1}^{\prime}-\left(a^{2}+b^{2}\right) q_{r}}{b^{2}-a^{2}}=-q_{r}$.

For $\frac{\left(\sigma_{\text {Oin }}^{\prime \prime}-\sigma_{\text {out }}^{\prime \prime}\right)}{\sigma_{\text {Oin }}^{\prime \prime}}=\frac{-3.189 \times 10^{7}-\left(-3.204 \times 10^{7}\right)}{-3.189 \times 10^{7}}=0.0047$ (as shown in Table 3). Therefore, it is reasonable that $q_{1}^{\prime}=q_{r}$ in the theoretical calculation.

In addition, the theoretical value of $q_{r}$ for the outer pipe entering full plasticity was $q_{r}=\sigma_{s 2} \ln \frac{c}{b}=3.207 \times 10^{7} \mathrm{~Pa}$, which was $1.6 \%$ different from the simulation value $\left(3.157 \times 10^{7} \mathrm{~Pa}\right)$. The value of $q_{1}$ for the outer pipe to enter the fully plastic regime was $q_{1}=\sigma_{s 1} \ln \frac{b}{a}=4.013 \times 10^{7} \mathrm{~Pa}$, which was $0.2 \%$ different from the simulation value $\left(4.004 \times 10^{7} \mathrm{~Pa}\right)$. Considering the numerical calculation got a discrete result, the time node corresponding to the selected stress value deviated slightly from the time node that we are interested in; the error was within the acceptable range.

\subsection{Influence of parameters on results of internal pressure method}

Setting $\sigma_{s 1}=\sigma_{s 2}=\sigma_{s}, E_{1}=E_{2}, v_{1}=v_{2}, b=c-t, a=$ $c-2 t, c^{*}=c / t$, ( $t$ is the thickness of the pipe wall) in Eq. (28), we obtain the following:

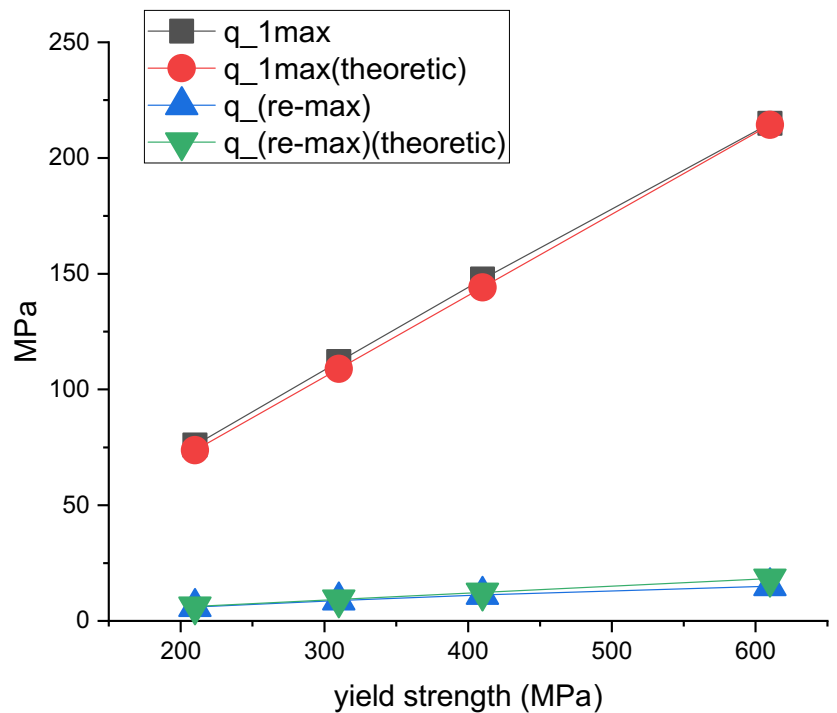

(b)

Fig. 6 Consistency between theory and numerical simulations when $\mathbf{a} \sigma_{s}$ was fixed at $210 \mathrm{MPa}$ and $\mathbf{b} c^{*}$ was fixed at 6.5 
$\frac{q_{r e-\max }}{\sigma_{s}}=\ln \frac{c^{*}}{c^{*}-1}-\frac{1}{B} \ln \frac{c^{*}}{c^{*}-2}$,

where

$B \equiv\left[\frac{2 c^{* 2}-2 c^{*}+1}{2 c^{*}-1}+\frac{2 c^{* 2}-6 c^{*}+5}{2 c^{*}-3}\right] \frac{2 c^{*}-3}{2 c^{* 2}-8 c^{*}+8}$.

Similarly, the inner wall pressure leading to the maximum residual stress is

$\frac{q_{1 \max }}{\sigma_{s}}=\ln \frac{c^{*}}{c^{*}-2}$.

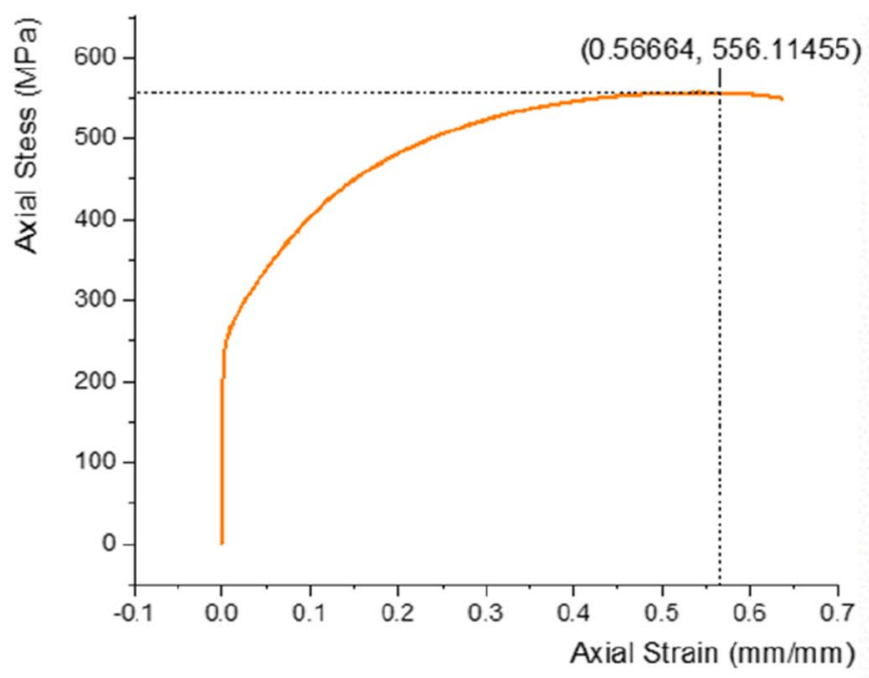

(a)

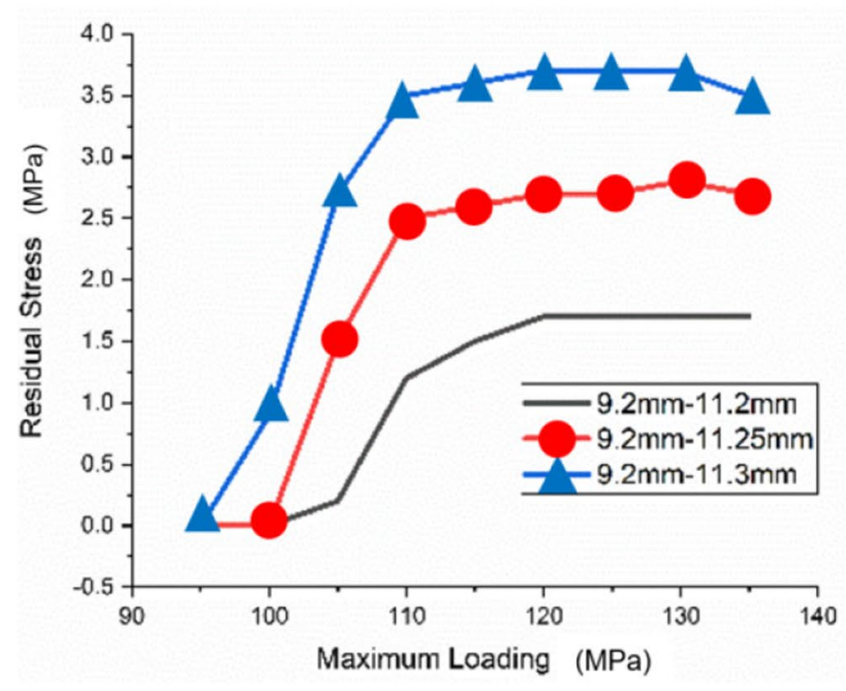

(c)
Figure 6a shows the relationships between $\frac{q_{r e-\max }}{\sigma_{s}}, \frac{q_{1 \max }}{\sigma_{s}}$, and $c^{*}$ at fixed $\sigma_{s}$, and Fig. 6b shows the relationships diagrams of $q_{r e-\max }, q_{1 \max }$, and $\sigma_{s}$ at fixed $c^{*}$. The theoretical results are compared with the numerical simulation results. An increase in $c^{*}$ caused decreases in $\frac{q_{r e-\max }}{\sigma_{s}}$ and $\frac{q_{1 \max }}{\sigma_{s}}$ when $\sigma_{s}$ was fixed, and the increase in $\sigma_{s}$ caused increases in $q_{r e-\max }$ and $q_{1 \max }$ when $c^{*}$ was fixed. Furthermore, the theory and numerical simulations were consistent. Thus, the theory can be used to predict the results of doublewalled pipes manufactured by the internal pressure method.

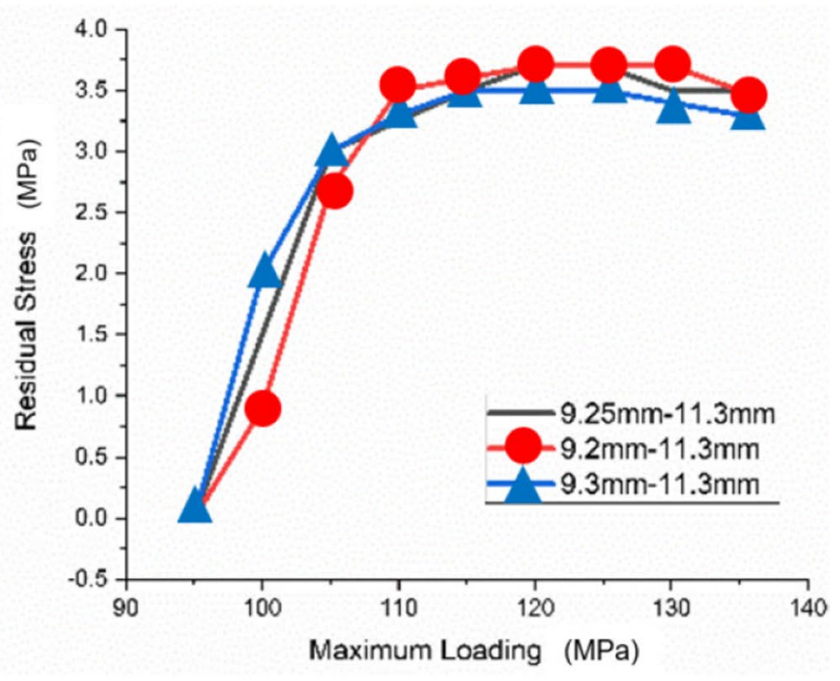

(b)

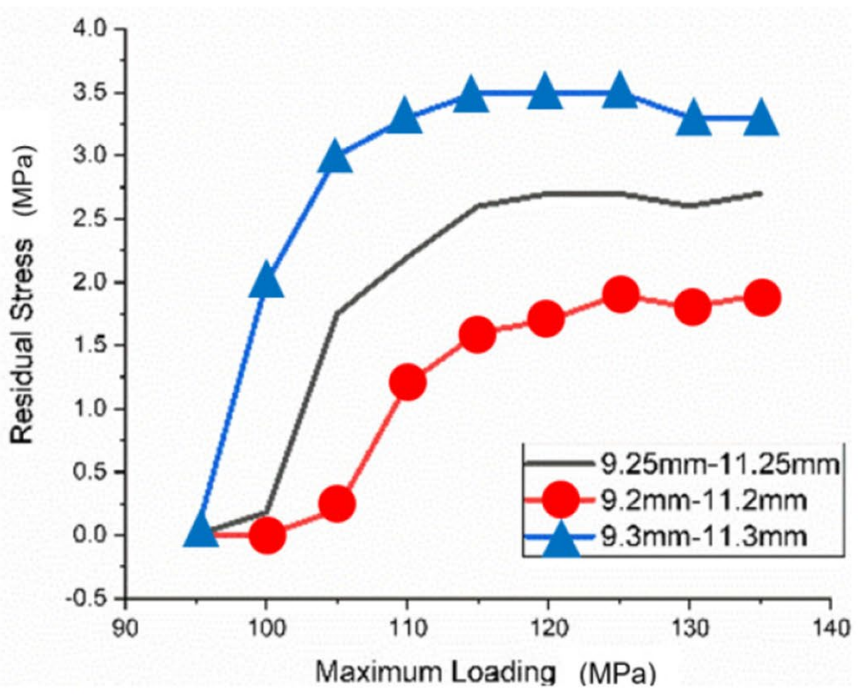

(d)

Fig 7 a Results of a simple tensile test of a real steel tube. Residual stress when the $\mathbf{b}$ inner radius of the inner pipe, $\mathbf{c}$ outer radius of the inner pipe, and $\mathbf{d}$ both the inner and outer radii of the inner pipe were varied 


\section{Adjustment for material with hardening effect}

The stress-strain curve of $316 \mathrm{~L}$ stainless steel obtained by a simple tensile test is shown in Fig. 7a. For materials with a hardening function $\sigma_{h}=\sigma_{h}\left(\varepsilon_{p}\right)$, Eq. (22) is written as

$q_{1}=q_{p}+q_{r}+q_{h}$

When the internal pressure $q_{p}=\sigma_{s 1} \ln \frac{b}{a}$ and the inner pipe was fully in the plastic regime, $q_{h}$ appeared during the period from the inner pipe entering the complete plastic regime to making contact with the outer pipe. $q_{h}$ is related to the hardening function and the distance between the inner and outer pipes, which is written as $q_{h}=q_{h}\left(\sigma_{h}, d\right)$. Figures $7 \mathrm{~b}$, c, and d show that the results of the simulation verified the influence of the spacing $d$ given by Eq. (35). The trend was roughly consistent with previously reported results [11].

Inequality (26) can be changed to the following:

$(B-1) q_{r}>q_{p}+q_{h}\left(\sigma_{h}, d\right)$.

For a material with a hardening function, the success of the internal expansion method will be influenced by the distance between the inner and outer pipes. As $q_{h}$ increases with $d$, inequality (38) becomes difficult to satisfy, so it will become more difficult to compress the two pipes with a larger gap. In the design and manufacturing process, this factor should be fully considered.

\section{Conclusion}

In this work, the mechanical extrusion process for manufacturing double-walled pipes was analyzed using the thickwalled assumption, ideal elastoplastic relationship, and Tresca yield condition. An analytical model of the doublewalled pipe was derived, and the criterion for manufacturing double-walled pipes was obtained. The residual stress between the inner and outer pipes is necessary for successful manufacturing, which can be predicted by theory. Numerical simulations were used to verify the accuracy of the theory.

In the manufacturing process using actual materials with hardening effects, the gap between the inner and outer layers needs to be considered because its influence on the residual stress is larger than that of other parameters. This work can provide a theoretical basis for the industrial manufacturing of double-walled pipes. The conclusions from the mold processing method analysis were similar to those of the theoretical analysis.

Based on actual production conditions, the assumption that for a wide enough space, the inner pipe is completely plastic when it contacts the outer pipe was proposed, and the reliability was verified. However, the theoretical calculations and numerical simulations in this work were based on quasistatic processes, so they are not valid for dynamic processes, such as in explosion-based methods. Furthermore, the deformation and phase transformations of steel crystals in the processing were not discussed in this paper, which will be a subject of future work.

Acknowledgements This work was supported by the Fundamental Research Funds for the Central Universities (WK2090050040) and the Major Program of the National Science Foundation of China (No.11890683). This work was jointly supported by the Strategic Priority Research Program of the Chinese Academy of Sciences (XDB22040402) and the National Natural Science Foundation of China (11525211). We acknowledge support from CAS Key Laboratory of Mechanical Behavior and Design of Materials (LMBD). We thank LetPub (www.letpub.com) for its linguistic assistance during the preparation of this manuscript.

Author contribution The complete theoretical analysis and numerical simulation of the process of preparing double-walled tube by internal pressure method are presented.

Funding Included in the Acknowledgements.

Data availability statement Some or all data, models, or code that support the findings of this study are available from the corresponding author upon reasonable request.

\section{Declarations}

Ethics approval and consent to participate Not applicable.

Conflict of interest The authors declare no competing interests.

Open Access This article is licensed under a Creative Commons Attribution 4.0 International License, which permits use, sharing, adaptation, distribution and reproduction in any medium or format, as long as you give appropriate credit to the original author(s) and the source, provide a link to the Creative Commons licence, and indicate if changes were made. The images or other third party material in this article are included in the article's Creative Commons licence, unless indicated otherwise in a credit line to the material. If material is not included in the article's Creative Commons licence and your intended use is not permitted by statutory regulation or exceeds the permitted use, you will need to obtain permission directly from the copyright holder. To view a copy of this licence, visit http://creativecommons.org/licenses/by/4.0/.

\section{References}

1. Rozzia D, Forgione N (2015) Experimental, investigation on powder conductivity for the application to double wall bayonet tube bundle steam generator. 24th International Conference on Nuclear Energy for New Europe (NENE)

2. Sato M, Patel MH, Trarieux F (2008) Static displacement and elastic buckling characteristics of structural pipe-in-pipe crosssections, structural engineering and mechanics 30:263-278

3. Bahman Z (2019) Application of heat pipes to fissionable nuclear reactor. https://doi.org/10.1007/978-3-030-05882-1_8 
4. Yican Wu, Minghuang W, Qunying $\mathrm{H}$ et al (2015) Research status and development prospect of lead - based reactors. Chin J Nucl Sci Eng 02:213-221

5. Shuang J (2015) Stress analysis of heat transfer pipe of steam generator in nuclear power plant. North China Electric Power University

6. Flatley T, Thursfield T (1984) Review of corrosion resistant coextruded tube development for power boilers. Conf Coatings and Bimetallics for Energ Sys Chem Process Environ South Carolina $18-22$

7. Haiyun C, Zhixi C (2006) Application and development of bimetallic composite pipe plastic forming technology. Process Equipment \& Piping 43:16-18

8. Delong J F, Teranishi H, YoshikaWa K et al (1984) Metallurgical examination after one year of service of experimental 17 - 14 CuMo I. D. /O. D. Chromized and TP310 Clad Tubing Installed in Eddystone unit No. 1. Conf Coatings and Bimetallics for Energ Sys Chem Process Environ South Carolina 62

9. Bogatov N A, Bogatov A, Salikhyanov D R (2018) New engineering solutions in the production of laminated composite pipes for the oil industry. In: Brebbia C, Connor J (eds). Progress in Mater Sci Eng 163-169

10. Taheri-Behrooz F, and Pourahmadi E (2018) Mutual effect of coriolis acceleration and temperature gradient on the stress and strain field of a glass/epoxy composite-pipe, applied mathematical modelling. 59:164-182

11. Kim JB, Park CG, Kim HW, Jeong JY (2013) Residual stress evaluation of a double wall tube for SFR steam generator. Korea Atomic Energy Research Institute 10:24-25
12. Xueshu W, Peining Li, Chaxiu G (2001) Hydraulic expansion device of stainless steel composite pipe. Mechanical Engineer 2:10-11

13. Jianbin Lv, Yan L, Huiguang G (1995) Mechanical analysis of double layer tube composite forming. Journal of Taiyuan Heavy Machinery Institute 16(2):103-108

14. Jia Jianbo Xu, Yan. (2009) Plastic forming process of bimetallic composite pipe. Journal of Beihua University: Natural Science Edition 10(3):279-284

15. McLean M (1985) Creep deformation of metal-matrix composites. Compos Sci Technol 23:37-52. https://doi.org/10.1016/02663538(85)90010-7

16. Kamal SM, Dixit US (2016) A study on enhancing the performance of thermally autofrettaged cylinder through shrink-fitting. J Manuf Sci Eng Trans ASME 138:1-5

17. Lu XF, Zheng X (2011) "Optimization of drawing parameters for 20/316L bimetal-lined pipe based on FEM simulation", Zhongguo Youse Jinshu Xuebao/Chinese. J Nonferrous Met 21:205-213

Publisher's Note Springer Nature remains neutral with regard to jurisdictional claims in published maps and institutional affiliations. 